\title{
Magnetohydrodynamic entry flow for a plane channel in an axial magnetic field
}

\author{
By GERALD P. D'ARCY AND PHILIP S. SCHMIDT
}

Department of Mechanical Engineering, University of Texas at Austin

(Received 18 November 1973 and in revised form 12 August 1976)

An integral solution is described for flow of an electrically conducting fluid in a plane channel in a magnetic field which is aligned with the direction of the mean flow. It is shown that the presence of the magnetic field retards the development of the velocity profile by producing Lorentz forces which oppose the movement of fluid from the viscous wall region to the core. Solutions are presented for the entry length as a function of the magnetic interaction parameter. Solutions are also given for the dependence of the frictional component of the pressure drop on the magnetic field strength. The transverse pressure gradient produced by Lorentz forces is discussed for a typical case.

\section{Introduction}

Because of its numerous applications to MHD power generation and pumping, the problem of flow of a conducting fluid in a magnetic field transverse to the mean velocity has received much attention in the literature since Hartmann introduced the fully developed solution in 1937 (see Brandt \& Gillis 1965; Shohet 1963; Maciulaitis \& Loeffler 1964; Goins 1965). Flows in which the magnetic field is oriented along the mean velocity axis have received relatively little attention. In the fully developed laminar flow, no Lorentz force is generated, since all velocities are parallel to the field; in the entry region of a channel, however, velocity components transverse to the mean flow do exist, and hence a magnetic body force is exerted on the fluid which tends to retard the growth of the viscous wall layers and hence lengthen the entry region.

Several investigators have examined the question of stability of MHD flows in axially directed fields. Stuart (1954) considered analytically the stability of plane Poiseuille flow in a longitudinal magnetic field. By determining the Reynolds number for neutral stability of the least stable mode of disturbance, he predicted the variation in transition Reynolds number and showed that it should increase with increasing Hartmann number.

Bader \& Carlson (1958) conducted experiments with mercury in a glass tube at Reynolds numbers up to 8000 and Hartmann numbers up to about 50. They measured the overall pressure drop in the tube and concluded that the magnetic field had little effect on transition. This conclusion was questioned, however, because the pressure taps were positioned such that it was impossible to distinguish hydrodynamic and magnetic entry effects from the developed flow 
region, and these entry effects may have masked any increment in the pressure drop caused by transition.

Globe (1961) also performed experiments in mercury, at Hartmann numbers up to 20 and Reynolds numbers up to 20000 . He also inferred the onset of transition from pressure-drop measurements, but placed the pressure taps well downstream of the entrance to the solenoid and thus was able to avoid the interpretation problem faced by Bader \& Carlson. His data clearly showed the effects of the longitudinal field on transition, and in fact were well correlated by Stuart's theory even though Globe's tests were run in a tube rather than a plane channel.

Fraim \& Heiser (1968) measured the pressure drop for mercury flow in tubes and in addition used a single hot wire spanning the tube diameter to make a gross measurement of the intermittency of the flow. This technique was valuable as a sensitive qualitative indicator of the onset of transition. The experiments covered Reynolds numbers up to 11000 and Hartmann numbers up to about 75. Fraim \& Heiser's conclusions differed markedly from those of Globe. Their results fell far below Stuart's theoretical predictions for plane channels, indicating either that the difference in geometry was significant or that the longitudinal field had much less of a stabilizing influence on the flow than had previously been supposed.

Branover (1967) performed similar stability experiments, extending the range of Reynolds and Hartmann numbers to 50000 and 360 , respectively. He further confirmed the stabilizing influence of the magnetic field on transition.

In all the above investigations, the emphasis was on stability of the flow rather than entry-region development. Fraim \& Heiser did suggest that MHD entryregion effects might have influenced their friction-factor data, but at the low magnetic interaction parameters studied, this effect would have been masked by other uncertainties in the experiment; as will be shown later in this paper, entryregion effects become significant only at much higher interaction parameters than those previously studied experimentally.

The motivation to analyse the entry-length problem in more detail arises from its possible application in the design of fusion reactors. In these reactors, liquidmetal coolants flow along magnetic field lines after entering the breeding blanket. Both the pressure drop and the heat-transfer characteristics of the flow will be affected by the lengthening of the entry region, and hence it is necessary to analyse magnetic field effects in order to estimate these design parameters. In this application, the fluid (say lithium) will have to cross magnetic field lines to enter the blanket, but will ultimately flow parallel to the field. Hartmann numbers of $10^{4}$ will be typical. Hartmann (1937) showed that for transverse fields the velocity profile gets progressively 'flatter' with increasing Hartmann number. For example, when $M=10$ the maximum velocity is only about $10 \%$ higher than the mean. It is reasonable, therefore, to make the idealization that the starting velocity profile for the axial-field portion of the flow in a fusion reactor will, for all practical purposes be uniform. It is further assumed that the flow will be incompressible and laminar; at the Reynolds numbers of interest, the magnetic field may be expected to suppress turbulence. The walls of the channel are assumed to be non-conducting.

An integral approach was selected for an attack on this problem. While some 


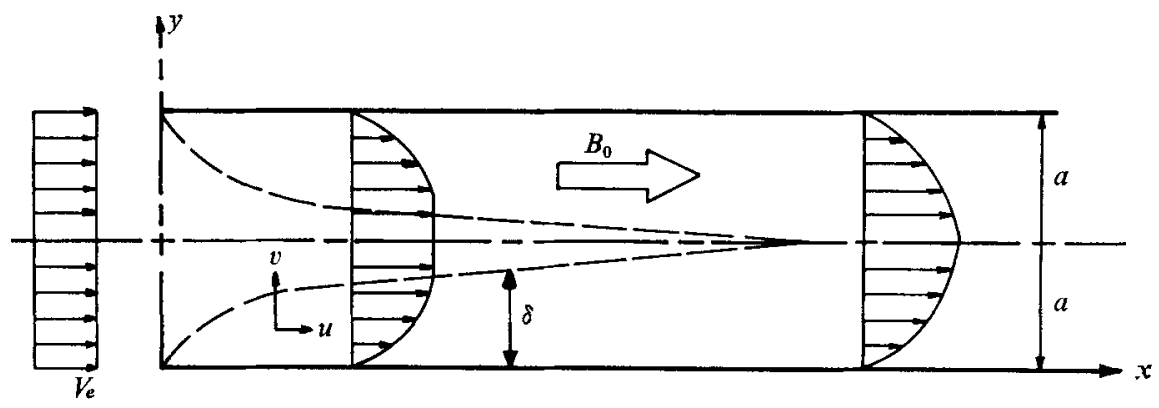

Figure 1. Channel configuration.

of the structure of the flow is lost in the integration process, the method does yield sufficiently detailed results to enable one to assess the desirability of a more complete solution, and to provide useful input to a design analysis.

\section{Analysis}

\section{Formulation}

Figure 1 illustrates the channel configuration and the co-ordinate system used. The velocity at the entrance is assumed to be uniform and of magnitude $V_{e}$. Viscous effects bring the fluid to rest at the wall, causing the formation of a region where the longitudinal velocity varies from zero at the wall to some nearly uniform value in the core of the channel. The depth $\delta$ of the boundary layer grows with distance along the channel until the two boundary layers eventually meet, marking the end of the entry region and the onset of fully developed flow. Since the longitudinal mass flux is less in the boundary layer than in the core region, continuity requires the generation of a transverse fluid velocity away from the walls. The transverse flux is responsible for two effects. First, the flux produces an increase in the 'uniform' longitudinal core velocity; second, the transverse component is normal to the applied magnetic field $B_{0}$ and the ensuing interaction results in a Lorentz force which generally opposes the growth of the boundary layer. Hence, unlike a normal boundary-layer problem, the $y$ momentum equation must be retained in the formulation.

The $x$ and $y$ momentum equations and the continuity equation may be written as

$$
\begin{gathered}
u \frac{\partial u}{\partial x}+v \frac{\partial u}{\partial y}=\frac{1}{\rho} X-\frac{1}{\rho} \frac{\partial P}{\partial x}+\nu\left(\frac{\partial^{2} u}{\partial x^{2}}+\frac{\partial^{2} u}{\partial y^{2}}\right) \\
u \frac{\partial v}{\partial x}+v \frac{\partial v}{\partial y}=\frac{1}{\rho} Y-\frac{1}{\rho} \frac{\partial P}{\partial y}+\nu\left(\frac{\partial^{2} v}{\partial x^{2}}+\frac{\partial^{2} v}{\partial y^{2}}\right) \\
\partial u / \partial x+\partial v / \partial y=0
\end{gathered}
$$

where $u$ and $v$ are the longitudinal and transverse velocities respectively, $x$ and $y$ are the co-ordinates shown in figure $1, P$ is the pressure, and $X$ and $Y$ are body forces per unit volume in the $x$ and $y$ directions respectively. 
The pressure may be eliminated from the first two equations by cross-differentiating them and subtracting (equivalent to taking the curl of the vector equation of motion). Rearrangement of terms yields the two-dimensional vorticity equation:

$$
u \frac{\partial \omega}{\partial x}+v \frac{\partial \omega}{\partial y}=\frac{1}{\rho}\left(\frac{\partial Y}{\partial x}-\frac{\partial X}{\partial y}\right)+v\left(\frac{\partial^{2} \omega}{\partial x^{2}}+\frac{\partial^{2} \omega}{\partial y^{2}}\right)
$$

where $\omega \equiv \partial v / \partial x-\partial u / \partial y$ is the $z$ component of the vorticity.

The Lorentz-force equation defines the electromotive body force:

$$
\mathbf{F}=X \hat{\mathbf{x}}+Y \hat{\mathbf{y}}=\rho_{c} \mathbf{E}+\mathbf{J} \times \mathbf{B},
$$

where $\rho_{c}$ is the local charge density. The current is related to the electric and magnetic fields through Ohm's law

$$
\mathbf{J}=\sigma(\mathbf{E}+\mathbf{V} \times \mathbf{B}) .
$$

In this study, the electric field is everywhere assumed to be zero. Assuming that the induced magnetic fields are negligible, the body force becomes

$$
X=0, \quad Y=-\sigma B_{0}^{2} v .
$$

Equations (4) and (7) may be combined and made dimensionless with the following parameters:

$$
u^{\prime}=u / V_{e}, \quad v^{\prime}=v / V_{e}, \quad x^{\prime}=x / a, \quad y^{\prime}=y / a,
$$

where $V_{e}$ is the entrance velocity or mean fluid velocity and $a$ is the channel half-width. Equation (4) then becomes

$$
u^{\prime} \frac{\partial \omega^{\prime}}{\partial x^{\prime}}+v^{\prime} \frac{\partial \omega^{\prime}}{\partial y^{\prime}}=N \frac{\partial v^{\prime}}{\partial x^{\prime}}+\frac{1}{R}\left(\frac{\partial^{2} \omega^{\prime}}{\partial x^{\prime 2}}+\frac{\partial^{2} \omega^{\prime}}{\partial y^{\prime 2}}\right)
$$

where $\omega^{\prime}=\partial v^{\prime}\left|\partial x^{\prime}-\partial u^{\prime}\right| \partial y^{\prime}, R=a V_{e} / \nu$ is the Reynolds number, and $N=\sigma B_{0}^{2} a /$ $\rho V_{e}$ is the magnetic interaction parameter.

Carrying out an order-of-magnitude analysis and neglecting second-order terms, (8) becomes

$$
u^{\prime} \frac{\partial^{2} u^{\prime}}{\partial x^{\prime} \partial y^{\prime}}+v^{\prime} \frac{\partial^{2} u^{\prime}}{\partial y^{\prime 2}}=N \frac{\partial v^{\prime}}{\partial x^{\prime}}+\frac{1}{R} \frac{\partial^{3} u^{\prime}}{\partial y^{\prime 3}}
$$

Application of the continuity equation at each cross-section yields a second governing equation:

$$
\int_{0}^{1} u^{\prime} d y^{\prime}=1
$$

Equation (9) may be integrated across the boundary layer as follows:

$$
\int_{0}^{\phi} u^{\prime} \frac{\partial^{2} u^{\prime}}{\partial x^{\prime} \partial y^{\prime}} d y^{\prime}+\int_{0}^{\phi} v^{\prime} \frac{\partial^{2} u^{\prime}}{\partial y^{\prime 2}} d y^{\prime}=N \int_{0}^{\phi} \frac{\partial v^{\prime}}{\partial x^{\prime}} d y^{\prime}+\frac{1}{R} \int_{0}^{\phi} \frac{\partial^{3} u^{\prime}}{\partial y^{\prime 3}} d y^{\prime}
$$

where $\phi$ is the non-dimensional boundary-layer thickness $\delta / a$. Assuming that $u^{\prime}=u_{0}$ and $\partial u^{\prime} / \partial y^{\prime}=\partial^{2} u^{\prime} / \partial y^{\prime 2}=0$ at $y^{\prime}=\phi$ and that $u^{\prime}=v^{\prime}=0$ at $y^{\prime}=0$, this integral equation becomes

$$
u_{0} \frac{d u_{0}}{d x^{\prime}}=N \int_{0}^{\phi} \frac{\partial v^{\prime}}{\partial x^{\prime}} d y^{\prime}-\frac{1}{R}\left(\frac{\partial^{2} u^{\prime}}{\partial y^{\prime 2}}\right)_{y^{\prime}=0},
$$

where $u_{0}$ is the dimensionless uniform core velocity. 
Equations (10) and (11) may be converted into ordinary differential equations using an assumed velocity profile. The parabolic profile represented by the equation

$$
u^{\prime}=\left\{\begin{array}{lll}
u_{0}\left[2\left(y^{\prime} / \phi\right)-\left(y^{\prime} / \phi\right)^{2}\right] & \text { when } y^{\prime}<\phi \\
u_{0} & \text { when } y^{\prime}>\phi
\end{array}\right\}
$$

was selected, since it gives the exact solution at both ends of the entry region, since the velocity vanishes at the wall and since the profile connects smoothly with the uniform core velocity at the edge of the boundary layer.

The continuity equation may be used to determine the corresponding transverse velocity profile:

or

$$
v^{\prime}=-\int_{0}^{y^{\prime}} \frac{\partial u^{\prime}}{\partial x^{\prime}} d y^{\prime}
$$

$$
v^{\prime}=\left\{\begin{array}{lll}
A^{\prime}\left(y^{\prime} \mid \phi\right)^{2}+B^{\prime}\left(y^{\prime} \mid \phi\right)^{3} & \text { when } y^{\prime}<\phi, \\
C^{\prime}\left(1-y^{\prime}\right) & \text { when } y^{\prime}>\phi,
\end{array}\right\}
$$

where

$$
\begin{gathered}
C^{\prime}=\frac{3}{(3-\phi)^{2}} \frac{d \phi}{d x^{\prime}}, \\
A^{\prime}=C^{\prime}(3-2 \phi), \quad B^{\prime}=-C^{\prime}(2-\phi) .
\end{gathered}
$$

Using the assumed profiles, (10) becomes

or

$$
\begin{gathered}
\int_{0}^{1} u^{\prime} d y^{\prime}=\int_{0}^{\phi} u^{\prime} d y^{\prime}+\int_{\phi}^{1} u^{\prime} d y^{\prime}=1, \\
u_{0} \int_{0}^{\phi}\left[2\left(\frac{y^{\prime}}{\phi}\right)-\left(\frac{y^{\prime}}{\phi}\right)^{2}\right] d y^{\prime}+u_{0} \int_{\phi}^{1} d y^{\prime}=1, \\
u_{0}\left(1-\frac{1}{3} \phi\right)=1 .
\end{gathered}
$$

Note that (14) may alternatively be derived from the requirement that $v$ vanishes at the centre-line (as it must because of symmetry). This means that application of the continuity equation to any flow cross-section also guarantees the satisfaction of the boundary condition $v=0$ at $y^{\prime}=1$.

The various terms of (11) may now be evaluated. Substitution yields

$$
\begin{aligned}
\int_{0}^{\phi} \frac{\partial v^{\prime}}{\partial x^{\prime}} d y^{\prime} & =\int_{0}^{\phi} \frac{\partial^{2}}{\partial x^{\prime 2}}\left(\int_{0}^{y^{\prime}} u^{\prime} d y^{\prime}\right) d y^{\prime} \\
& =\frac{\phi^{2}}{4} \frac{d^{2} u_{0}}{d x^{\prime 2}}-\frac{\phi}{3}\left(\frac{d \phi}{d x^{\prime}}\right)\left(\frac{d u_{0}}{d x^{\prime}}\right)-\frac{\phi}{6} u_{0} \frac{d^{2} \phi}{d x^{\prime 2}}+\frac{u_{0}}{6}\left(\frac{d \phi}{d x^{\prime}}\right)^{2}
\end{aligned}
$$

and

$$
\partial^{2} u^{\prime} / \partial y^{\prime 2}=-2 u_{0} / \phi^{2} \text {. }
$$

Equation (11) then becomes

$$
u_{0} \frac{d u_{0}}{d x^{\prime}}-N\left[\frac{\phi^{2}}{4} \frac{d^{2} u_{0}^{\prime}}{d x^{\prime 2}}-\frac{\phi}{3}\left(\frac{d \phi}{d x^{\prime}}\right)\left(\frac{d u_{0}}{d x^{\prime}}\right)-\frac{\phi}{6} u_{0} \frac{d^{2} \phi}{d x^{\prime 2}}+\frac{u_{0}}{6}\left(\frac{d \phi}{d x^{\prime}}\right)^{2}\right]-\frac{2 u_{0}}{R \phi^{2}}=0
$$

Equations (14) and (15) may be combined to give an ordinary differential equation in one unknown. Equation (14) may be rearranged as

$$
u_{0}=3 /(3-\phi)
$$


and differentiated to obtain relations between the derivatives:

$$
\begin{gathered}
\frac{d u_{0}}{d x^{\prime}}=\frac{3}{(3-\phi)^{2}}\left(\frac{d \phi}{d x^{\prime}}\right) \\
\frac{d^{2} u_{0}}{d x^{\prime 2}}=\frac{3}{(3-\phi)^{2}}\left(\frac{d^{2} \phi}{d x^{\prime 2}}\right)+\frac{6}{(3-\phi)^{3}}\left(\frac{d \phi}{d x^{\prime}}\right)^{2}
\end{gathered}
$$

Equations (14)-(17) may be combined as follows:

$$
\frac{36}{3-\phi} \frac{d \phi}{d x^{\prime}}-\frac{24(3-\phi)}{R \phi^{2}}=N\left[(6-5 \phi) \phi \frac{d^{2} \phi}{d x^{\prime 2}}-\frac{\left(12 \phi^{2}-24 \phi+18\right)}{3-\phi}\left(\frac{d \phi}{d x^{\prime}}\right)^{2}\right] .
$$

Introducing the dimensionless co-ordinate $\eta=x / a R=x^{\prime} / R$ gives

$$
\left(\frac{36}{3-\phi}\right) \frac{d \phi}{d \eta}-\frac{24(3-\phi)}{\phi^{2}}=\frac{N}{R}\left[(6-5 \phi) \phi \frac{d^{2} \phi}{d \eta^{2}}-\frac{\left(12 \phi^{2}-24 \phi+18\right)}{3-\phi}\left(\frac{d \phi}{d \eta}\right)^{2}\right] .
$$

This means that all flows with the same $N / R$ may be represented by a single solution of (19). Rearrangement of (19) produces the equation to be solved numerically:

$$
\frac{d^{2} \phi}{d \eta^{2}}=\left\{\frac{\left(12 \phi^{2}-24 \phi+18\right)}{3-\phi}\left(\frac{d \phi}{d \eta}\right)^{2}+\frac{R}{N}\left[\left(\frac{36}{3-\phi}\right) \frac{d \phi}{d \eta}-\frac{24(3-\phi)}{\phi^{2}}\right]\right\} /(6-5 \phi) \phi .
$$

Neither (19) nor (20) appears to be soluble by direct analytical techniques, and even numerical techniques present problems in the region very near the origin. Equation (20) clearly shows a singularity in $d^{2} \phi / d \eta^{2}$ at $\eta=0$. This difficulty may be circumvented by using a power-series approximation in the neighbourhood of the origin. Using this technique, initial conditions are obtained at some point in the neighbourhood of the origin and the solution is then continued using (20), which is well behaved away from the origin.

The following truncated series was used for this approximation:

At $\eta=0, \phi=0 ;$ therefore

$$
\begin{gathered}
\phi=A+B \eta^{n} . \\
\phi=B \eta^{n} .
\end{gathered}
$$

If $n<1$, all derivatives of $\phi$ will have the expected singularities at the origin. Substitution of this series into (19) yields $B=(24 R / N)^{\frac{1}{4}}$ and $n=\frac{1}{2}$,

or

$$
\phi=(24 R / N)^{\frac{1}{1}} \eta^{\frac{1}{2}} \text {. }
$$

Before describing the numerical approach, it should be observed that (19) may be solved analytically when $N=0$. Equation (19) then becomes

$$
d \phi / d \eta=2(3-\phi)^{2} / 3 \phi^{2}
$$

which may be written in terms of $u_{0}$ using (14):

$$
d u_{0} / d \eta=2 u_{0}^{2} / 9\left(u_{0}-1\right)^{2} .
$$

Rearranging and integrating this equation and setting $\phi=1$ (or $u_{0}=\frac{3}{2}$ ) yields a value for the length of the entry region with no magnetic field:

$$
x_{e 0} / a=R / 9 \cdot 9 \text {. }
$$


The approximate core velocities obtained in this analysis for $N=0$ compared well with two finite-difference solutions (Bodoia \& Osterle 1961; Brandt \& Gillis 1965) and a perturbation solution obtained by Schlichting (1934). The solutions are not coincident, but are in reasonable agreement considering their approximate nature. Bodoia \& Osterle's solution predicts an infinite entry region with the centre-line velocity asymptotically approaching the steady-state value. The integral solution predicts a finite entry region. Bodoia \& Osterle's solution shows that $96 \%$ of the fully developed velocity has been reached at a point corresponding to the end of the approximate entry length determined in the present study. Comparable agreement was obtained for computed pressure distributions for the above solutions.

\section{Solution for $N \neq 0$}

For the case where $N$ is not equal to zero, (20) was solved numerically as follows. Initial values of $\phi$ and $\phi^{\prime}$ were determined for the first increment using the powerseries approximation. A Runge-Kutta integration was used to obtain the next three points, and remaining values were computed using an Adams-Bashforth predictor and an Adams-Moulton corrector scheme.

Determination of the pressure drop is somewhat more involved than in the non-magnetic case. The magnetic body force causes the pressure to vary across the channel; therefore a mean pressure is defined as

$$
\bar{P}=\frac{1}{a} \int_{0}^{a} P d y .
$$

Application of the momentum theorem from the channel wall to the centre-line, and from the entrance to an axial point $x$, gives

$$
\rho \int_{0}^{a} u^{2} d y-\rho a V_{e}^{2}=\left(P_{0}-\bar{P}\right) a-\int_{0}^{x} \tau d x,
$$

where $\tau$ is the local wall shear stress and $P_{0}$ is the entrance pressure. Rearrangement yields

$$
\frac{P_{0}-\bar{P}}{\rho V_{e}^{2}}=\int_{0}^{1}\left(\frac{u}{V_{e}}\right)^{2} d\left(\frac{y}{a}\right)+\int_{0}^{x / a} \frac{\tau}{\rho V_{e}^{2}} d\left(\frac{x}{a}\right)-1 .
$$

The integrals may be simplified using the assumed velocity distribution:

$$
\begin{gathered}
\int_{0}^{1}\left(\frac{u}{V_{e}}\right)^{2} d\left(\frac{y}{a}\right)=u_{0}^{2}\left(1-\frac{7 \phi}{15}\right), \\
\int_{0}^{x / a} \frac{\tau}{\rho V_{e}^{2}} d\left(\frac{x}{a}\right)=2 \int_{0}^{\eta} \frac{u_{0}}{\phi} d \eta .
\end{gathered}
$$

By substitution, the pressure-drop equation then becomes

$$
\frac{\Delta \widetilde{P}}{q}=2 u_{0}^{2}\left(1-\frac{7 \phi}{15}\right)+4 \int_{0}^{\eta} \frac{u_{0}}{\phi} d \eta-2
$$

wher $q=\frac{1}{2} \rho V_{e}^{2}$ is the dynamic pressure. 


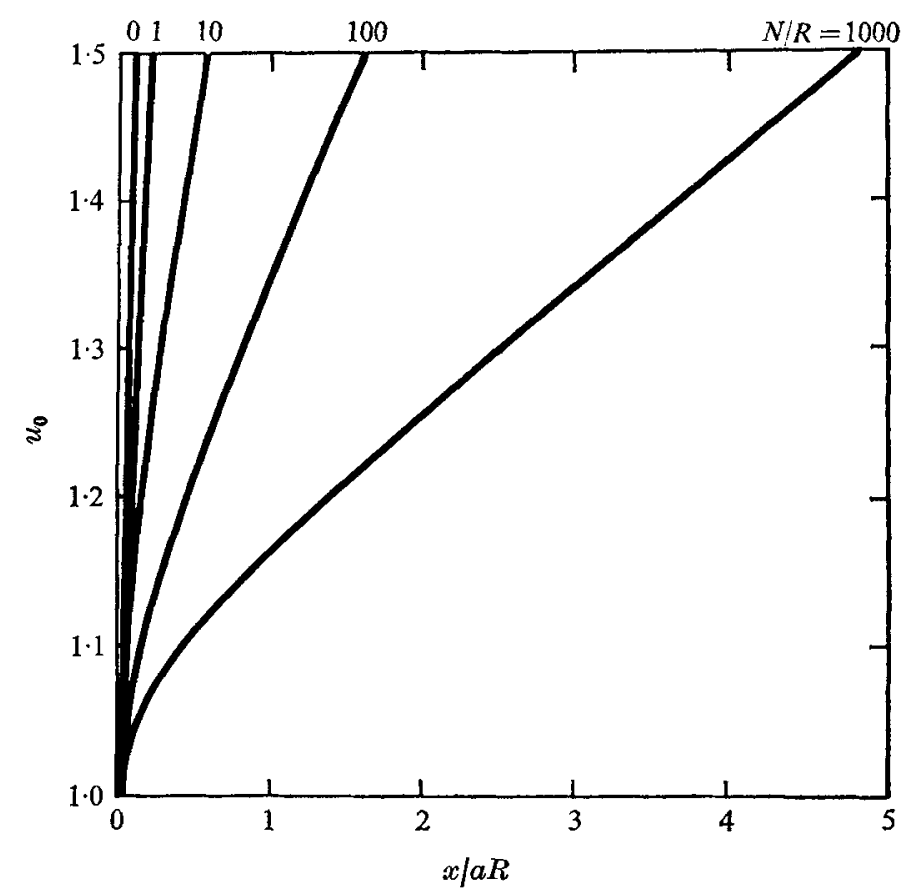

Figure 2. Effect of magnetic field upon the developing core velocity of channel entry flows.

The power-series approximation for $\phi$ was used to evaluate the integral term in the neighbourhood of the origin. That is,

$$
\int_{0}^{\eta} \frac{u_{0}}{\phi} d \eta=\int_{0}^{\eta_{1}}\left(\frac{3}{3-\phi}\right) \frac{d \eta}{\phi}+\int_{\eta_{1}}^{\eta} \frac{u_{0}}{\phi} d \eta
$$

where $\eta_{1}$ is the end of the first increment. Then

and

$$
\begin{gathered}
d \eta=2 \phi B^{-2} d \phi \\
\int_{0}^{\eta_{1}}\left(\frac{3}{3-\phi}\right) \frac{d \eta}{\phi}=\frac{6}{B^{2}} \int_{0}^{\phi_{1}} \frac{d \phi}{3-\phi}=\frac{6}{B^{2}} \ln u_{01},
\end{gathered}
$$

where $\phi_{1}$ and $u_{01}$ are the non-dimensional boundary-layer thickness and centreline velocity respectively at $\eta_{1}$. Finally, the pressure-drop equation becomes

$$
\frac{\Delta \bar{P}}{q}=2 u_{0}^{2}\left(1-\frac{7 \phi}{15}\right)-2+\frac{24}{B^{2}} \ln u_{01}+4 \int_{\eta_{1}}^{\eta} \frac{u_{0}}{\phi} d \eta
$$

The trapezoidal rule was used to evaluate the integral in this expression.

\section{Results of the analysis for $N \neq 0$}

\section{Entry length and core velocity}

Figure 2 illustrates the effect of the magnetic field on the development of the core velocity in the entry region. This figure shows the dimensionless centre-line velocity plotted against dimensionless distance along the channel for a range of 
MHD entry flow for a plane channel

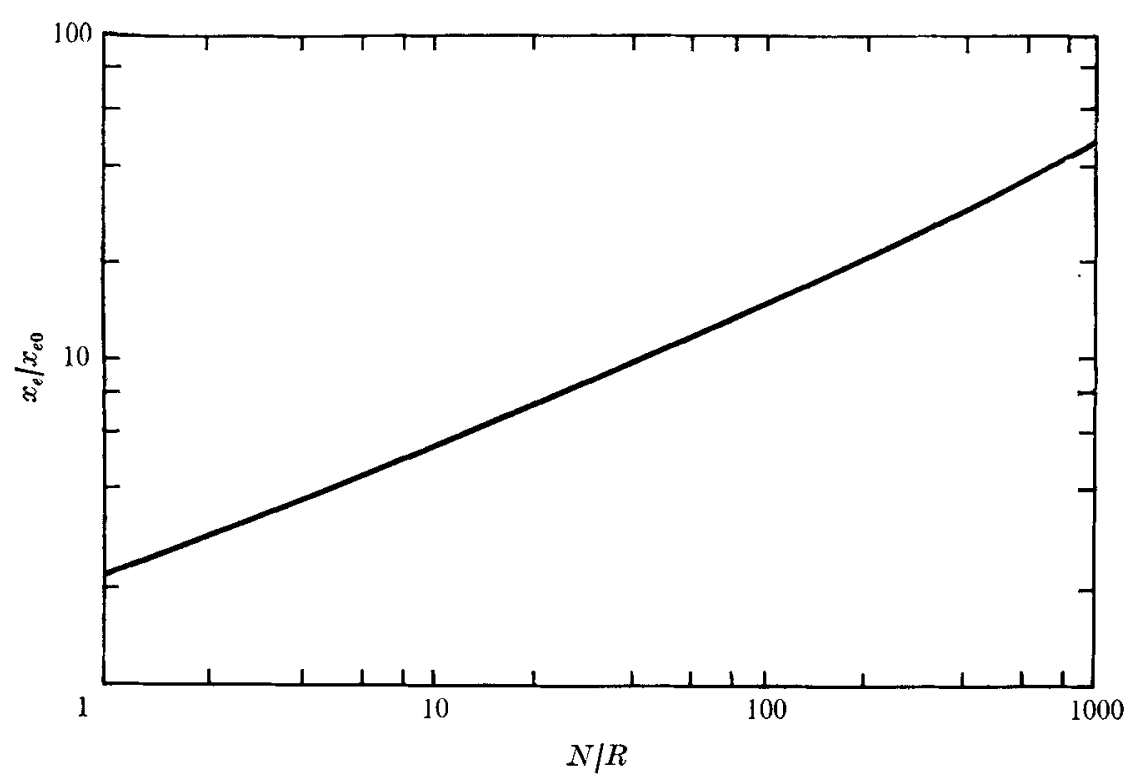

FIGURE 3. Ratio of total entry length $x_{e}$ to non-magnetic entry length $x_{e 0} v s . N / R$.

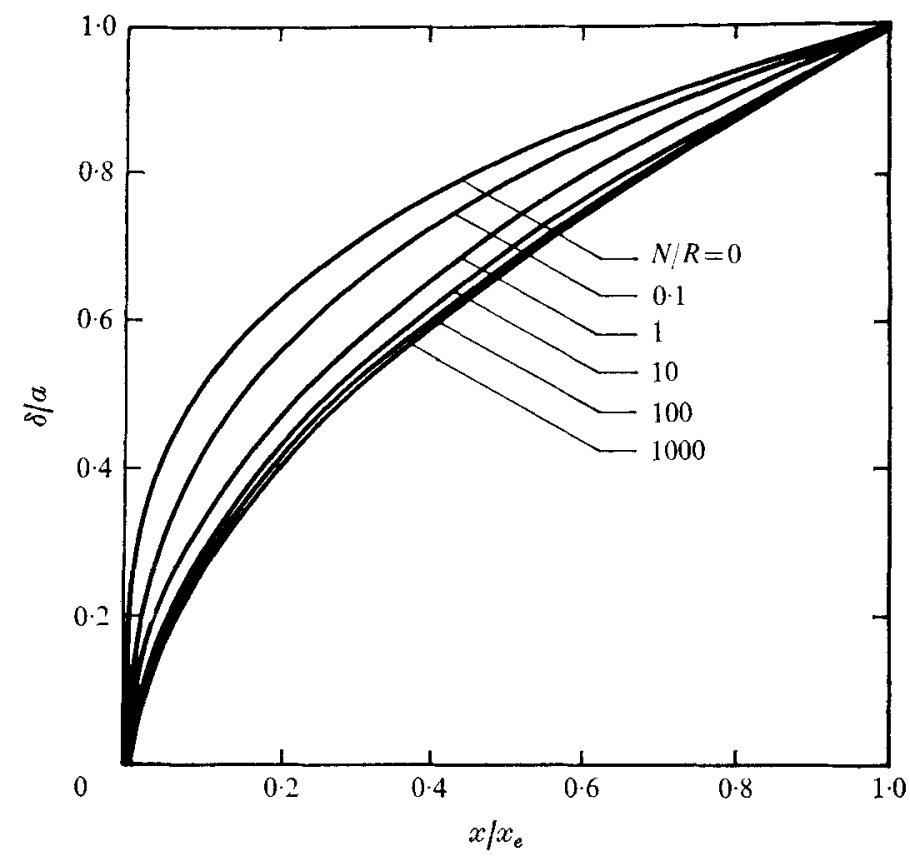

Figure 4. Normalized boundary-layer profiles for channel entry flows. 


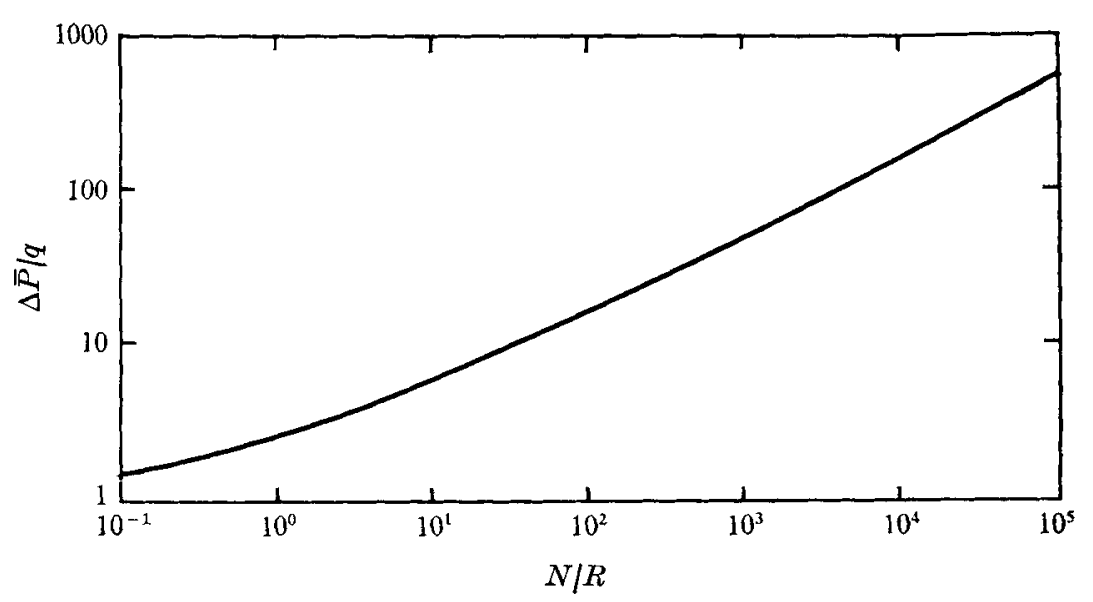

Figure 5. Total pressure drop through entry region.

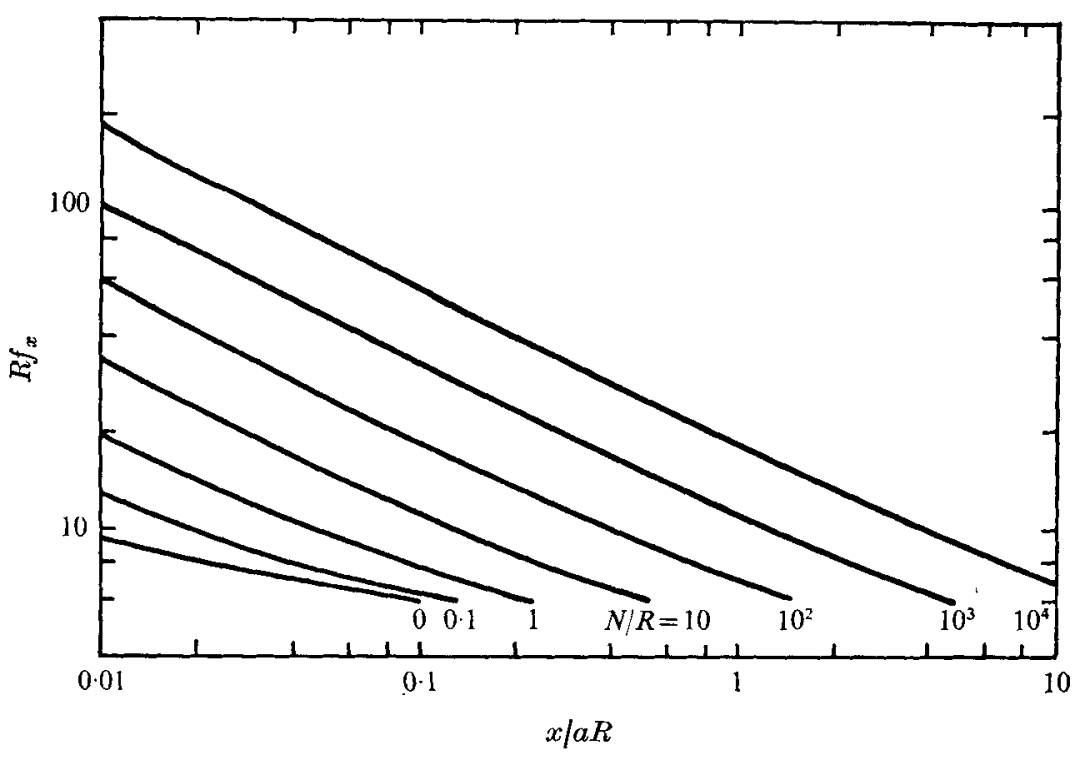

Figure 6. Local friction factor for channel entry flows.

$N / R$. Recall that $N$ is proportional to the square of the magnetic field strength. The end of the channel entry region is identified by the point where the dimensionless velocity is $\mathbf{1} \cdot \mathbf{5}$. The MHD entry regions develop more slowly than those for non-magnetic flows as expected.

Figure 3 shows the ratio of the total MHD entry length $x_{e}$ to the hydrodynamic (non-magnetic) entry length $x_{e 0}$, plotted against the ratio $N / R$. When $N / R=$ 1000 , the entry length is increased to nearly fifty times the non-magnetic value. By way of example, imagine a $100 \mathrm{kG}$ field acting on lithium flowing into a channel 3 in. wide. If the Reynolds number is 10000 , the ratio $N / R$ will be $18 \cdot 9$. Then, according to figure 3 , the channel entry length would be $900 \mathrm{ft}$, compared 


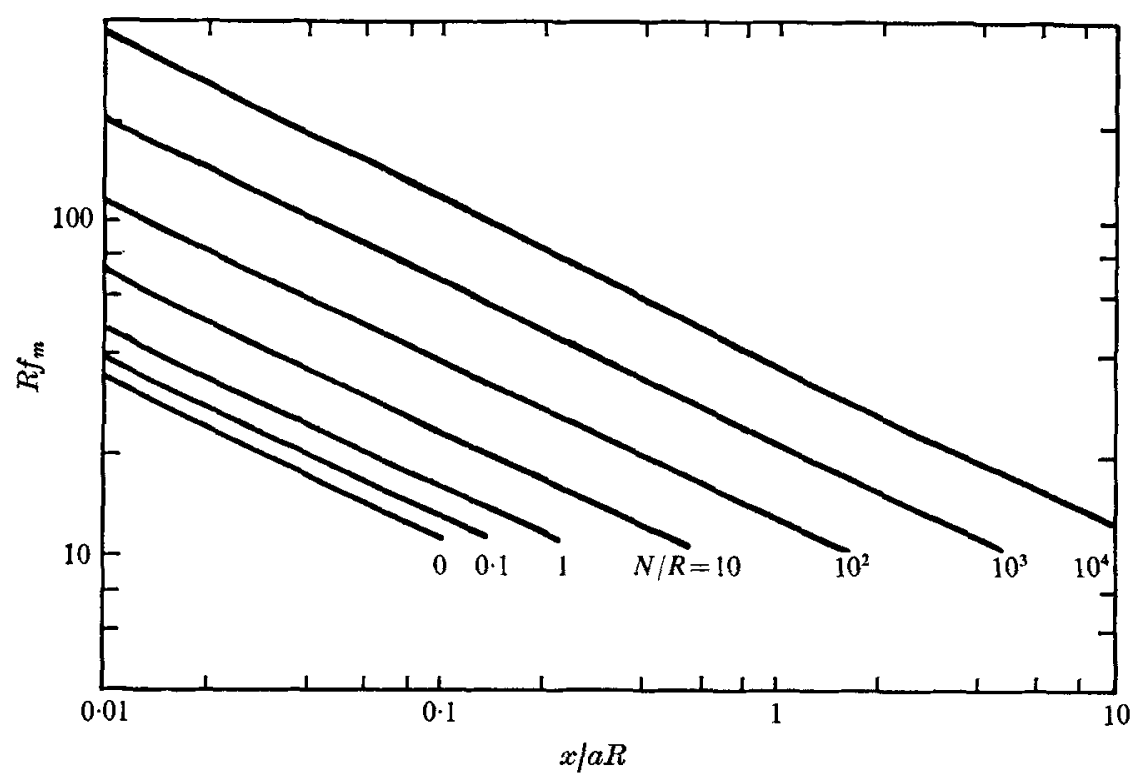

Fiaure 7. Mean friction factor for channel entry flows.

with about $125 \mathrm{ft}$ in the non-magnetic case (assuming a laminar non-magnetic flow). In the absence of the magnetic field, the flow at this Reynolds number would of course be turbulent and the non-magnetic entry length would be considerably shorter than $125 \mathrm{ft}$.

Figure 4 shows the normalized boundary-layer thickness as a function of axial location. For large values of $N / R$ the growth rate appears to approach a limiting value.

\section{Pressure drop}

Figure 5 illustrates the effect of increasing magnetic field on the total pressure drop, including the momentum change, through the entry region. The mean pressure drop, where mean pressure is defined as

$$
\bar{P}=\frac{1}{a} \int_{0}^{a} P d y,
$$

has been made dimensionless with the dynamic pressure $q=\frac{1}{2} \rho V_{e}^{2}$. The entrylength pressure drop for $N / R=1000$ is nearly fifty times the non-magnetic pressure drop.

Information on the total pressure drop through the entry region is of limited value from the practical standpoint, since the entry regions are so long that in many practical situations fully developed flow will never be attained. Figure 6 gives the local friction factor, which is defined in terms of the local shear stress at the wall

$$
\tau=f_{x}\left(\frac{1}{2} \rho V_{e}^{2}\right) .
$$

These curves may be integrated to obtain the wall drag due to friction in the entry region. This loss, combined with momentum changes associated with changes in 


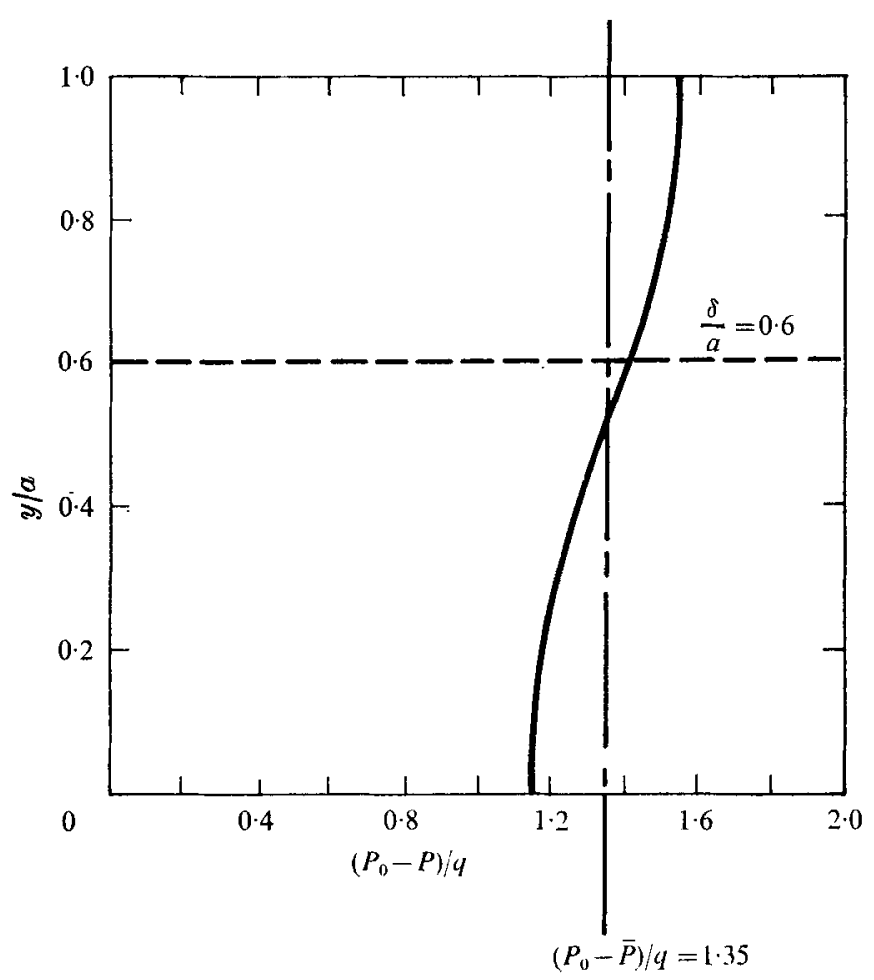

Figure 8. Typical transverse pressure distribution for channel entry flows. $x / a R=0 \cdot 075, N / R=1$.

the velocity distribution, gives the pressure drop up to a particular location. The local friction factor clearly increases with magnetic field strength.

Figure 7 gives mean friction factors which may be used to compute the mean frictional pressure drop up to any point within the entry region (i.e. not including momentum changes). This drop may be computed as

$$
\Delta P_{f}=f_{m}\left(\frac{1}{2} \rho V_{e}^{2}\right)(x / a) \text {. }
$$

As stated earlier, the pressure varies in the transverse $(y)$ direction owing to the magnetic body force, which is related to the transverse velocity by

$$
\partial P / \partial y=-N v \rho V_{e} / a \text {. }
$$

Figure 8 illustrates the significance of the cross-channel pressure variation including the momentum change, for a typical case. This figure shows the pressure defect for $N / R=1$ and $\phi=0 \cdot 6$. Half the change in the core velocity has occurred at this point and the mean pressure drop is $1.35 q$. At the wall the pressure drop is $1 \cdot 17 q$, i.e. the wall pressure is $0 \cdot 18 q$ greater than the mean pressure; the centre-line pressure drop is $1.55 q$, i.e. the centre-line pressure is $0.20 q$ less than the mean. Hence wall pressure measurements made during an experiment would tend to be higher than mean pressures in the entry region. 


\section{Conclusions}

It has been shown that, while the presence of an axial magnetic field will not affect the fully developed laminar flow behaviour of a conducting fluid, body forces induced by transverse velocities may have a significant effect in the entry region. For high magnetic fields, the entry length may be extended by more than an order of magnitude, and the pressure drop for this region will consequently be increased substantially. The cross-channel magnetic body force is associated with a pressure gradient normal to the flow axis in the entry region. The analysis presented in this paper is of an approximate nature, and hence it is recommended that the numerical results, particularly with regard to the cross-channel pressure gradient, be used with care. The integral method, however, has become well accepted for entry-length and pressure-drop estimates, and the authors feel that these figures should be adequate for preliminary design purposes.

\section{REFERENCES}

Bader, M. \& CarLson, W. C. A. 1958 Measurement of the effect of an axial magnetic field on the Reynolds number of transition in mercury flowing through a glass tube. N.A.C.A. T'ech. Note, no. 4274.

Bodoia, J.R. \& Osterle, J. F. 1961 Finite difference analysis of plane Poiseuille and Couette flow developments. Appl. Sci. Res. A 10, 265-276.

Brandt, A. \& Gillis, J. 1965 Magnetohydrodynamic flow in the inlet region of a straight channel, AFOSR Rep. no. 65-2213, part 2, ASTIA AD 628180.

Branover, G. G. 1967 Suppression of turbulence in pipes with transverse and longitudinal magnetic fields. Magnetohydrodyn. 3, 156-175.

Fraim, F. W. \& Heiser, W. H. 1968 Effect of a strong longitudinal magnetic field on the flow of mercury in a circular tube. J. Fluid Mech. 33, 397-413.

GLore, S. 1961 Effect of a longitudinal magnetic field on pipe flow of mercury. A.S.M.E. J. Heat Transfer, 83, 445-453.

Gorss, E. E. 1965 The influence of the initial velocity profile on MHD flow development in the entrance region of a parallel plate channel. M.Sc. thesis, AFIT, WrightPatterson AFB, Ohio.

Hartmann, J. V.L. 1937 Theory and experimental flow of plasma in a magnetic field (mercury flows). $K$. danske vindensk. Selsk. Mat.fys. Medd. 15, 6-7.

Maciulaitis, A. \& Lofffler, A. L. 1964 A theoretical investigation of MHD channel entrance flows. A.I.A.A. J. 2, 2100-2103.

Schlichting, H. 1934 Laminare Kanaleinlaufstromung. Z. angew. Math. Mech. 14, 368-373.

SHонет, J. L. 1963 Entry problems in electrohydrodynamics. Phys. Fluids, 6, 1524-1525.

Stuart, J. T. 1954 On the stability of viscous flow between parallel planes in the presence of a co-planar magnetic field. Proc. Roy. Soc. A 221, 189-206. 